\title{
Wachsende Ungleichheit lässt Ökonomen kalt
}

\author{
Seit geraumer Zeit ist die wachsende Ungleichheit das Kardinalproblem unserer Gesellschaft, \\ wenn nicht der gesamten Menschheit. Während daraus im globalen Maßstab Krisen und \\ Kriege resultieren, die wiederum größere Migrationsbewegungen nach sich ziehen, sind \\ hierzulande sozialer Zusammenhalt und repräsentative Demokratie bedroht. Gleichwohl \\ haben sich die meisten deutschen Ökonomen nie ernsthaft mit dem Problem der Ungleichheit \\ auseinandergesetzt und nach Möglichkeiten für eine Lösung gesucht. Stattdessen haben sie \\ das Problem ignoriert oder seine Bedeutung negiert bzw. relativiert.
}

\begin{abstract}
Für Jahrhunderte wurde die sozioökonomische Ungleichheit als naturgegeben oder gottgewollt hingenommen und selten hinterfragt. Seit der europäischen Aufklärung, den bürgerlichen Revolutionen und dem Sturz zahlreicher Monarchien bedarf Ungleichheit der politischen Legitimation, sofern sie weder natürlich noch sachlich, etwa durch Ausbildungs-, Wissens- und Kompetenzunterschiede zwischen bestimmten (Gruppen von) Personen, begründet ist. Vorher wurden Ungleichheitsstrukturen auch von den negativ Betroffenen im Wesentlichen ohne kollektiven Widerstand akzeptiert. Nur besonders gebildete Minderheiten begaben sich schon zu jener Zeit auf die Suche nach den Ursachen der ökonomischen, sozialen und politischen Ungleichheit, die weit verbreitet und extrem stark ausgeprägt war.
\end{abstract}

\section{Vom Ursprung der Ungleichheit}

Jean-Jacques Rousseau beteiligte sich 1754 an einer Ausschreibung der Académie des Dijon, deren Preisfrage lautete, welchen Ursprungs die Ungleichheit unter den Menschen und ob diese durch das Naturrecht autorisiert sei. Ohne damit die ausgelobte Goldmedaille im Wert von 30 Pistolen zu gewinnen, gab Rousseau darauf die folgende, mittlerweile klassisch zu nennende Antwort: „Der erste, der ein Stück Land eingezäunt hatte und es sich einfallen ließ zu sagen: dies ist mein und der Leute fand, die einfältig genug waren, ihm zu glauben, war der wahre Gründer der bürgerlichen Gesellschaft. Wie viele Verbrechen, Kriege, Morde, wie viel Not und Elend und wie viele Schrecken hätte der-

Prof. Dr. Christoph Butterwegge lehrte von 1998 bis 2016 Politikwissenschaft in Köln und ist Mitglied der Forschungsstelle für interkulturelle Studien (FiSt) an der Universität zu Köln. jenige dem Menschengeschlecht erspart, der die Pfähle herausgerissen oder den Graben zugeschüttet und seinen Mitmenschen zugerufen hätte: ,Hütet euch, auf diesen Betrüger zu hören; ihr seid verloren, wenn ihr vergeßt, daß die Früchte allen gehören und die Erde niemandem.“"1

Niemand hat die negative Wirkung des Privateigentums (an Grund und Boden), ausgehend von einem Naturzustand der sozialen Gleichheit aller Menschen, jemals prägnanter charakterisiert als Rousseau. Der Wirtschaftssoziologe Johannes Berger behauptete 250 Jahre später, dass Rousseau durch die Problematisierung der Landnahme sein Hauptaugenmerk auf die marktmäßige Beschränktheit des Bodens gerichtet habe, der (im Unterschied zum Kapital) nicht vermehrt werden kann. Demnach hätte Rousseau erkannt, dass Ungleichheit auf Einhegungen, also auf Zugangsbeschränkungen, beruhe: „Daher wäre es verfehlt, das genaue Gegenteil von Zugangsbeschränkungen, die freie Konkurrenz, für die Entstehung, den Fortbestand und die Zunahme ökonomischer Ungleichheiten verantwortlich zu machen, so populär diese Auffassung heute auch sein mag."

Rousseau führte sämtliche Ausprägungen der Ungleichheit (Rang, Macht, persönliches Verdienst und Reichtum) auf den Reichtum als erste Stufe bzw. Urform der Ungleichheit zurück. $^{3}$ Offenbar hielt Rousseau die Einkommens- und Vermögensverteilung für das zentrale Kriterium zur Beurteilung der sozialen Egalität in einem Land und den Gegen-

(C) Der/die Autor(en) 2020. Open Access: Dieser Artikel wird unter der Creative Commons Namensnennung 4.0 International Lizenz (https:// creativecommons.org/licenses/by/4.0/deed.de) veröffentlicht.

Open Access wird durch die ZBW - Leibniz-Informationszentrum Wirtschaft gefördert.

1 J.-J. Rousseau: Diskurs über die Ungleichheit/Discours sur l'inégalité. Kritische Ausgabe des integralen Textes, Paderborn 2019, S. 173.

2 J. Berger: Über den Ursprung der Ungleichheit unter den Menschen. Zur Vergangenheit und Gegenwart einer soziologischen Schlüsselfrage, in: Zeitschrift für Soziologie, 33. Jg. (2004), H. 5, S. 356.

3 Vgl. J.-J. Rousseau, a. a. O., S. 255; J. Berger, a. a. O., S. 358. 
satz zwischen Arm und Reich für die ausschlaggebende Scheidelinie in jeder auf privatem Eigentum basierenden Gesellschaft. Wenn man überdies bedenkt, dass Rousseau im Ancien Regime lebte, also nicht in einer kapitalistischen Industriegesellschaft, war sein Fokus auf die Landnahme und den privaten Grundbesitz als Quellen der sozioökonomischen Ungleichheit mehr als verständlich. Marxens Kritik der politischen Ökonomie knüpfte ungefähr 100 Jahre später bruchlos daran an, indem sie die gesellschaftliche Arbeitsteilung, das Privateigentum an Produktionsmitteln und die Aneignung des Mehrprodukts durch eine privilegierte Minderheit für die Klassenspaltung verantwortlich machte.

\section{Armut im Überfluss}

Georg Friedrich Wilhelm Hegel hielt die soziale Ungleichheit bereits für ein Schlüsselthema der Gesellschaftstheorie. In seiner 1820 erschienenen Rechtsphilosophie behandelte er Armut und Reichtum nicht als Strukturgegensätze, sondern als zusammengehörige, die bürgerliche Gesellschaft verbindende Pole. Auch benutzte Hegel mit Blick auf die Arbeiterschaft schon damals den Klassenbegriff, um sie als das negative Ergebnis der Ungleichheit verkörpernde Bevölkerungsgruppe zu kennzeichnen. Hegel beklagte die Konzentration des Reichtums in wenigen Händen. ${ }^{4}$ Für den Berliner Philosophen als führenden Denker seiner Zeit wurden Arme erst durch die innere Empörung gegenüber den Reichen, der Gesellschaft und der Regierung zum „Pöbel“, wie er auch ihre Arbeitsscheu und ihren Drang kritisierte, die Subsistenz als Recht geltend zu machen: „Gegen die Natur kann kein Mensch ein Recht behaupten, aber im Zustande der Gesellschaft gewinnt der Mangel sogleich die Form eines Unrechts, was dieser oder jener Klasse angetan wird. Die wichtige Frage, wie der Armut abzuhelfen sei, ist eine vorzüglich die modernen Gesellschaften bewegende und quälende." ${ }^{5}$ Weiter stellte Hegel an gleicher Stelle fest, „daß bei dem Übermaße des Reichtums die bürgerliche Gesellschaft nicht reich genug ist, d.h. an dem ihr eigentümlichen Vermögen nicht genug besitzt, dem Übermaße der Armut und der Erzeugung des Pöbels zu steuern.“

Kurz nach der Jahrtausendwende hat der Soziologe Oskar Negt unter Berufung auf Hegel den sich zuspitzenden Wi-

4 „Durch die Verallgemeinerung des Zusammenhangs der Menschen durch ihre Bedürfnisse und der Weisen, die Mittel für diese zu bereiten und herbeizubringen, vermehrt sich die Anhäufung der Reichtümer - denn aus dieser gedoppelten Allgemeinheit wird der größte Gewinn gezogen - auf der einen Seite, wie auf der andern Seite die Vereinzelung und Beschränktheit der besonderen Arbeit und damit die Abhängigkeit und Not der an diese Arbeit gebundenen Klasse, womit die Unfähigkeit der Empfindung und des Genusses der weiteren Freiheiten und besonders der geistigen Vorteile der bürgerlichen Gesellschaft zusammenhängt.“ G. F. W. Hegel: Grundlinien der Philosophie des Rechts, in: ders.: Werke, Bd. 7, Frankfurt a. M. 1970, S. 389.

5 Ebenda, S. 390 derspruch thematisiert, dass der moderne Kapitalismus ein Übermaß an Ressourcen hervorbringt, ohne die soziale Ungleichheit beseitigen zu können, und in diesem Zusammenhang gegenwartsbezogen konstatiert: „Wenn eine Arbeitsund Erwerbsgesellschaft, die in beschleunigtem Tempo Reichtum produziert, mit dem Armutsproblem nicht fertig werden kann, dann ist das ein Zeichen, daß eine historisch entstandene Verteilungsordnung überholt ist."6

\section{Die deutsche „Normalität der Ungleichheit“}

In der „alten“ Bundesrepublik suchten namhafte Soziologen wie Helmut Schelsky, Ralf Dahrendorf oder Ulrich Beck die als marxistische Signalbegriffe verpönten Termini „Klasse“ bzw. „Klassengesellschaft“ durch weniger brisante Kategorien zu ersetzen, ihren Wortsinn zu entschärfen oder sie auf andere Weise ideologisch zu entsorgen. ${ }^{7}$ Hingegen sparten die führenden Vertreter der Wirtschaftswissenschaft das Thema „Ungleichheit“ meist ganz aus. „Die meisten Ökonomen machen sich über Ungleichheit keine Gedanken, da sie aus ,Grenzproduktivität‘ oder Investitionen in Humankapital hervorgeht: ein vermeintlich gerechtes und zwangsläufiges Ergebnis wirtschaftlichen Wachstums."8 Nur heterodoxe Ökonomen betrachten die soziale Ungleichheit als relevantes Problem und streben ihre Verringerung an, indem privater Reichtum durch Staatsinterventionen umverteilt wird. Heiner Flassbeck und Paul Steinhardt halten die in den meisten Ländern zunehmende Ungleichheit für „die große gesellschaftliche Herausforderung unserer Zeit"9, deren Wurzel sie in den Arbeitsbeziehungen sehen: „Die entscheidende Frage ist, in welcher Weise die Machtverhältnisse am ,Arbeitsmarkt' die Entwicklung der Einkommen prägen, wie man das politisch verhindern kann und warum man es aus wirtschaftlichen Gründen verhindern muss." 10

Der ökonomische Mainstream legitimiert die zunehmende Ungleichheit, indem er sie zur notwendigen Voraussetzung eines produktiven Wirtschafts- und Gesellschaftssystems bzw. zu einer Triebkraft des wissenschaftlich-technischen Erkenntnisfortschritts emporstilisiert. In einer Marktgesellschaft, die durch Ökonomisierung, Privatisierung und Kommerzialisierung fast aller Lebensbereiche gekennzeichnet ist, gilt wirtschaftliche bzw. soziale Ungleichheit als normal. Vertreter der ökonomischen Klassik und Neoklassik erklärten sie überwiegend sogar für nützlich und notwendig, damit die als „Wirtschaftsstandort“ betrachtete Volkswirt-

6 O. Negt: Arbeit und menschliche Würde, Göttingen 2001, S. 240.

7 Vgl. hierzu C. Butterwegge: Die zerrissene Republik. Wirtschaftliche, soziale und politische Ungleichheit in Deutschland, Weinheim, Basel 2020, S. $67 \mathrm{ff}$.

8 M. Burawoy: Public Sociology. Öffentliche Soziologie gegen Marktfundamentalismus und globale Ungleichheit, Weinheim, Basel 2015, S. 191

9 H. Flassbeck, P. Steinhardt: Gescheiterte Globalisierung. Ungleichheit, Geld und die Renaissance des Staates, Berlin 2018, S. $151 \mathrm{f}$.

10 Ebenda. 
schaft erfolgreich sein kann. „Die Ökonomen haben sich vorrangig mit der Frage befaßt, wie eine Gesellschaft inren Wohlstand steigern kann. Die Frage der Verteilungsgerechtigkeit hielten sie demgegenüber für zweitrangig."11 Beispielhaft dafür stehen Koryphäen des Fachs wie Adam Smith, Friedrich August von Hayek und Milton Friedman. ${ }^{12}$ Zu fragen ist freilich, was es Armen nützt, in einem reichen Land zu leben, das sie mit Almosen abspeist. Die soziale Ungleichheit wird mit individuellen Leistungsunterschieden erklärt, weshalb sich Arme für ihr geringes Einkommen und mangelndes Vermögen rechtfertigen müssen. Reinhard Kreckel hat die Leistungsideologie als wichtigstes Mittel zur Legitimation von sozialer Ungleichheit in fortgeschrittenen Staatsgesellschaften bezeichnet und die „meritokratische Triade "13 von Bildungsabschluss, beruflichem Rang und Geldeinkommen - in der genannten Reihenfolge - problematisiert. Es ist ein meritokratischer Mythos, dem heute immer weniger Glauben geschenkt wird, dass Reichtum auf Leistung und Armut auf Leistungsverweigerung beruht.

\section{Kuznets lag falsch}

Ausgesprochen beruhigend wirkte die These von Simon Kuznets, wonach die sozioökonomische Ungleichheit während der Entwicklung eines Landes zunächst ansteigt und danach wieder sinkt. ${ }^{14}$ Die berühmte Kuznets-Kurve verläuft in einem Bogen von unten nach oben und wieder zurück, beschreibt sie doch, was der Harvard-Professor osteuropäischer Herkunft aufgrund seiner akribischen Längsschnittuntersuchung von Einkommensteuererklärungen und seiner Schätzung des jeweiligen Nationaleinkommens entdeckt zu haben glaubte. Mittlerweile ist die von Kuznets umgekehrtem und abgeflachtem „U“ ausgehende Faszination jedoch ebenso verblasst wie die Hoffnung darauf, dass sich die Einkommensungleichheit in den reichen Ländern des globalen Nordens reduziert. Mittlerweile kritisieren Ökonomen aus aller Welt die wachsende Verteilungsschieflage bei Einkommen und Vermögen. Der Ökonom Branko Milanović stellte 2016 fest: „Die unbestreitbare Zunahme der Ungleichheit in den Vereinigten Staaten, Großbritannien und sogar in einigen einigermaßen egalitären Ländern wie Schweden und Deutschland ist mit der

11 K. Methfessel, J. M. Winterberg: Der Preis der Gleichheit. Wie Deutschland die Chancen der Globalisierung verspielt, Düsseldorf, München 1998, S. 79.

12 Vgl. C. Butterwegge, B. Lösch, R. Ptak: Kritik des Neoliberalismus, Wiesbaden 2017.

13 „Die Qualifikation eines Individuums soll in eine entsprechende berufliche Position konvertierbar sein, die berufliche Position soll mit einem ihr angemessenen Einkommen ausgestattet sein - so will es die Leistungsideologie." Vgl. R. Kreckel: Politische Soziologie der sozialen Ungleichheit, Frankfurt a. M., New York 2004, S. 97.

14 Vgl. S. Kuznets: Economic Growth and Income Inequality, in: The American Economic Review, 45. Jg. (1955), H. 1, S. 1 ff.
Kuznets-Hypothese schlicht nicht vereinbar." ${ }^{\text {"15 }}$ Der französische Ökonom Thomas Piketty erklärte die Wiederzunahme der sozioökonomischen Ungleichheit in den meisten Industriestaaten seit den 1980er Jahren damit, dass die Kapitalrendite $(r)$ die Wachstumsrate von Wirtschaft und Einkommen (g) für längere Zeit überstieg. Mit seiner Formel $r>g$ hat Piketty zwar kein allgemein gültiges Verteilungsgesetz entdeckt, aber eine wichtige Tendenzaussage getroffen. Hieraus resultiert laut Piketty eine starke Triebkraft des modernen Kapitalismus zur wachsenden Ungleichheit, die inn zu folgender Prognose unter gleichen politischen Rahmenbedingungen veranlasste: „Wenn die Kapitalrendite dauerhaft höher ist als die Wachstumsrate von Produktion und Einkommen, was bis zum 19. Jahrhundert der Fall war und im 21. Jahrhundert wieder zur Regel zu werden droht, erzeugt der Kapitalismus automatisch inakzeptable und willkürliche Ungleichheiten, die das Leistungsprinzip, auf dem unsere demokratischen Gesellschaften basieren, radikal infrage stellen. ${ }^{* 16}$

Der britische Wirtschaftshistoriker Richard Wilkinson und die Epidemiologin Kate Pickett haben anhand einer Fülle empirischer Daten aus vielen Ländern belegt, dass die Mitglieder einer Gesellschaft umso gesünder sind, je gleicher deren materieller Reichtum unter innen verteilt ist. ${ }^{17}$ Von namhaften deutschen Ökonomen wird Ungleichheit aber immer noch ignoriert, negiert oder naturalisiert. Um die Jahrtausendwende, als der Marktradikalismus grassierte, avancierte Ungleichheit zum volkswirtschaftlichen Heilmittel, an dem Deutschland, damals irrigerweise als „kranker Mann Europas“ apostrophiert, genesen sollte. So konstatierten Klaus Methfessel und Jörg Winterberg, dass mehr Beschäftigung nur mit mehr Ungleichheit zu erreichen sei. ${ }^{18}$ An derselben Stelle beklagten die beiden Journalisten den angeblichen „Gleichheitswahn der beiden Volksparteien“, übersahen oder unterschlugen aber völlig, dass alle Bundesregierungen unter Führung der CDU/CSU bzw. der SPD durch gezielte Entlastung der Reichen ${ }^{19}$ einerseits sowie stärkere Belastung der Armen (steigende Mehrwertsteuer) andererseits systematisch die sozioöko-

15 B. Milanović: Die ungleiche Welt. Migration, das Eine Prozent und die Zukunft der Mittelschicht, Berlin 2016, S. 56.

16 T. Piketty: Das Kapital im 21. Jahrhundert, München 2014, S. 13 f.

17 „Ungleichverteilung führt zu geringerer Lebenserwartung, sie führt auch zu geringerem Geburtsgewicht und höherer Säuglingssterblichkeit.“ Vgl. R. Wilkinson, K. Pickett: Gleichheit ist Glück. Warum gerechte Gesellschaften für alle besser sind, Berlin 2010, S. 101.

18 „Der internationale Vergleich macht deutlich, daß es nur die beiden Alternativen gibt: entweder größere Ungleichheit bei den Löhnen und mehr Jobs oder größere Gleichheit und mehr Arbeitslose." Vgl. K. Methfessel, J. M. Winterberg. a.a. O., S. 77.

$19 \mathrm{Zu}$ nennen sind Abschaffung der Börsenumsatz- und Gewerbekapitalsteuer, Verzicht auf die Erhebung der Vermögensteuer, mehrfache Senkung des Spitzensteuersatzes und der Körperschaftsteuer sowie Einführung der Abgeltungssteuer auf Kapitalerträge und immer großzügigere Ausnahmeregelungen für Firmenerben bei der Erbschaftsteuer. 
nomische Ungleichheit gefördert haben. Wie die Bundesregierung im Vierten Armuts- und Reichtumsbericht selbst dokumentierte, verfügten die reichsten $10 \%$ der Bevölkerung im Jahr 1998, als das Buch von Methfessel und Winterberg erschien, über $45 \%$ des privaten Nettovermögens; 2003 waren es bereits $49 \%$ und 2008 sogar fast $53 \%$. Dagegen musste sich die ärmere Hälfte der Bevölkerung von 1998 bis 2003 mit $3 \%$ und 2008 mit bloß noch $1 \%$ begnügen. ${ }^{20}$ Diese gesellschaftlichen Polarisierungstendenzen waren weniger der wachsenden Dynamik des Weltmarkts geschuldet als den Privatisierungs- bzw. Deregulierungsentscheidungen, die Globalisierung, Modernisierung sowie Individualisierung flankierten und forcierten. Ein neoliberales Verständnis von Politik förderte die Polarisierung.

\section{Marktgläubigkeit statt Gesellschaftskritik}

Hans-Werner Sinn, seinerzeit Präsident des ifo Instituts für Wirtschaftsforschung, propagierte um die Jahrtausendwende eine Lohnsenkung bis zu einem Drittel sowie eine Senkung der Kapital- und Gewinnsteuern, erwähnte die daraus zwangsläufig resultierende größere Ungleichheit aber mit keinem Wort. ${ }^{21}$ Überhaupt kümmern sich nur wenige Ökonomen hierzulande um die Ungleichheit, behandeln dieses Thema vielmehr stiefmütterlich, wie DIW-Präsident Marcel Fratzscher zu Recht bemängelt. ${ }^{22}$ Fratzscher, der sein Buch „Verteilungskampf" genannt hat, scheut allerdings vor der logischen Schlussfolgerung einer Umverteilung des privaten Reichtums zurück und beschränkt sich im Wesentlichen auf die Forderung nach einem besseren Bildungssystem. Es soll mehr Chancengleichheit ermöglichen, die Hans-Olaf Henkel allen Ernstes längst verwirklicht sieht: „Jeder kann Unternehmer werden, und ebenso kann jeder Unternehmer, wenn er schlecht wirtschaftet, im Handumdrehen zum Hartz-IV-Empfänger werden.“"23

Friedrich Merz, früher Vorsitzender der CDU/CSU-Bundestagsfraktion und (noch) Aufsichtsratsvorsitzender des Vermögensverwalters BlackRock in Deutschland, erklärte die materielle Ungleichheit für nützlich und lehnte ihre Minderung ab, weil die Marktwirtschaft davon lebe und sie notwendigerweise „aus dem freien Wettbewerb um die besten Ideen, Innovationen und Produkte"24 hervorgehe. Merz hielt den Ruf nach mehr Gleichheit daher für verantwortungslos und im Ergebnis sogar für totalitär: „Es

20 Vgl. Bundesministerium für Arbeit und Soziales (Hrsg.): Lebenslagen in Deutschland. Der Vierte Armuts- und Reichtumsbericht der Bundesregierung, Bonn, März 2013, S. 465.

21 Vgl. H.-W. Sinn: Ist Deutschland noch zu retten? München 2003.

22 Vgl. M. Fratzscher: Verteilungskampf. Warum Deutschland immer ungleicher wird, München 2016, S. 249.

23 H.-O. Henkel: Der Kampf um die Mitte. Mein Bekenntnis zum Bürgertum, München 2007, S. 174.

24 Vgl. F. Merz: Mehr Kapitalismus wagen. Wege zu einer gerechten Gesellschaft, München, Zürich 2008, S. 28 f. ist die Forderung nach immer mehr materieller Gleichheit, die, ernst genommen, in Planwirtschaft und sozialistischer Diktatur mündet."25 Totalitär ist aber eher der doktrinäre Ausschluss gesellschaftlicher Entwicklungsalternativen, der verteilungspolitisch bedeutet, die Konzentration des Reichtums in wenigen Händen zu perpetuieren. Wer die materielle Ungleichheit auf diese Weise hypostasiert und Gleichheit nicht einmal zu denken wagt, zementiert den sozioökonomischen Status quo und handelt damit gegen jenen Teil der Bevölkerung, der kein nennenswertes Vermögen, aber ein hohes Armutsrisiko hat.

\section{Ökonomen glorifizieren Ungleichheit}

Während die Philosophie, die Theologie und alle übrigen Sozialwissenschaften heute weitgehend dem Ideal der Gleichheit verpflichtet sind, akzeptiert die Ökonomie mit der Koexistenz von massenhafter Armut und einem sich in wenigen Händen konzentrierenden Reichtum eine Form der Ungleichheit, die ihre führenden Repräsentanten sogar als Voraussetzung der Freiheit glorifizieren. Ungleichheit wird auf die Natur zurückgeführt, mit ihr gerechtfertigt und zur anthropologischen Konstante erklärt. Typisch dafür ist die Position von Michael Hüther und von Thomas Straubhaar, die sozioökonomische Ungerechtigkeit kurzerhand als subjektives Gefühl einer irregeleiteten Bevölkerungsmehrheit abtun. ${ }^{26}$ Der frühere Bundesbanker und Berliner Finanzsenator Thilo Sarrazin stellte die Ungleichheit ebenfalls als zwangsläufiges Resultat einer unterschiedlichen genetischen Ausstattung der Individuen hin. In seinem Bestseller „Deutschland schafft sich ab" geht er von der Überlegung aus, dass die soziale Ungleichheit auf unterschiedlichen Eigenschaften, Fähigkeiten und Fertigkeiten der Menschen beruht: „Wer schön oder intelligent ist, hat andere Chancen als jemand, der hässlich oder dumm ist. Und diese ungleiche Ausgangslage produziert unablässig Ergebnisse, die noch ungleicher sind." ${ }^{27}$

Natürlich sind Menschen weder biologisch noch sozial gleich, unterscheiden sich vielmehr im Alter, im Geschlecht, in der Größe, im Gewicht, im Körperbau, in der Haut-, Haar- und Augenfarbe sowie der Physiognomie voneinander - um nur einige phänotypische bzw. somatische Erkennungsmerkmale zu nennen -, aber auch im Hinblick auf ihre genetischen Dispositionen, Fähigkeiten und Fertigkeiten sowie in Bezug darauf, wo sie wohnen, in

25 Ebenda, S. 30.

26 „Ungleichheit ist Ausdruck der Unterschiedlichkeit der Menschen hinsichtlich ihrer Fähigkeiten, Begabungen und Präferenzen. Diese Ungleichheit einebnen zu wollen kann sich nur eine ideologisch verklemmte, ja totalitäre Politik erdreisten.“ Vgl. M. Hüther, T. Straubhaar: Die gefühlte Ungerechtigkeit. Warum wir Ungleichheit aushalten müssen, wenn wir Freiheit wollen, Berlin 2009, S. 322.

27 T. Sarrazin: Deutschland schafft sich ab. Wie wir unser Land aufs Spiel setzen, München 2010, S. 129. 
welchem Haushaltstyp und in welcher Familienform sie leben, welchen Beruf sie ausüben, ob sie Hobbys haben, ob sie regelmäßig Sport treiben usw. Die zuletzt genannten Eigenschaften der Individuen beruhen meist auf persönlichen Entscheidungen, die sie mehr oder weniger freiwillig getroffen haben. Es handelt sich hierbei um spezifische Formen der Ungleichheit, die nicht schicksalhaft vorbestimmt oder von der Natur bedingt sind, sondern individuell und selbstgewählt.

Jene verbreitete Ungleichheit, um die es in diesem Text geht, ist hingegen systemisch begründet, ökonomisch determiniert und in Gestalt von großen Bevölkerungsgruppen, Schichten oder Klassen sozial strukturiert; sie liegt also weder im eigenen Ermessen, noch ist sie einer natürlichen Ungleichheit der von ihr betroffenen Personen geschuldet. Vielmehr handelt es sich um eine Form der Ungleichheit, die politisch organisiert, legitimiert und perpetuiert, im Fall eines grundlegenden Systemwandels bzw. -wechsels aber restrukturiert oder destruiert wird.

\section{Bedarfs- oder leistungsgerecht?}

Während früher die Bedarfs- und die Verteilungsgerechtigkeit als konstitutive Leitwerte im Mittelpunkt der Diskussion standen, bestimmen heute Begriffe wie „Leistungsgerechtigkeit“, „Teilhabegerechtigkeit“, „Chancengerechtigkeit“ und "Generationengerechtigkeit“ die öffentliche Debatte. Sie tragen ebenfalls dazu bei, dass die sozioökonomische Ungleichheit von den meisten Bürgern als normal betrachtet und akzeptiert wird. Soll den oben genannten Zerfallsprozessen entgegengewirkt, Armut bekämpft und Reichtum beschränkt werden, muss nicht bloß die Sozialstruktur verändert, sondern auch das Bewusstsein für Polarisierungstendenzen und die mit innen verbundenen sozialen Probleme und die daraus erwachsenden Gerechtigkeitsdefizite geschärft werden.

Die wirtschaftliche Ungleichheit erklärt Verwerfungen unterschiedlicher Art. Denn sie strahlt auf die übrigen Gesellschaftsbereiche aus. Bei der Ungleichheit handelt es sich nie um ein rein ökonomisches Problem. Vielmehr existieren mannigfache Wechselwirkungen zwischen den verschiedenen Lebensbereichen. Die wirtschaftliche Ungleichheit manifestiert sich hauptsächlich in sozialer Ungleichheit. Da beide nur schwer voneinander zu trennen sind, bietet sich der Begriff „sozioökonomische Ungleichheit" als die präzisere Alternative geradezu an. Ökonomische und soziale Ungleichheit führen zwangsläufig auch zu politischer Ungleichheit, selbst dann, wenn es sich nicht - wie in den USA, wo großer politischer Einfluss und hohe Staatsämter mittlerweile hauptsächlich extrem reichen Menschen vorbehalten bleiben - um eine Plutokratie oder eine Präsidialdemokratie mit plutokratischen Zügen handelt.
Ökonomen möchten die Gleichheit auf die politische Sphäre beschränkt wissen, von ihrem eigenen Gegenstandsbereich - der Wirtschaft und der Gesellschaft - aber möglichst fernhalten. Man kann die Ökonomie deshalb als die Wissenschaft der Ungleichheit bezeichnen. Vielleicht ist allerdings eher von einer Marktideologie oder von einem Marktfundamentalismus statt von einer Wissenschaft zu sprechen, wenn Alternativen wie Gleichheit und Ungleichheit nicht einmal gedanklich zugelassen, sondern dogmatisch ausgeschlossen werden.

\section{Versuche der Beschönigung und Beschwichtigung}

Wenn sich ökonomische, soziale, ökologische und politische Probleme mehren, wächst in jeder Gesellschaft das Verlangen, die Augen davor zu verschließen, die mit innen verbundenen Gefahren zu verdrängen und sich das Debakel schönzureden. Ausgerechnet im Vorfeld schwerer ökonomischer oder ökologischer Krisen und in gesellschaftlichen Umbruchsituationen hat eine Beschönigungs- und Beschwichtigungsliteratur regelrecht Hochkonjunktur. Momentan greift sie den Klimawandel genauso wie den Themenkreis "Rechtsextremismus und Gewalt" oder das Problemfeld „Armut und sozioökonomische Ungleichheit“ auf. Der Soziologe Martin Schröder etwa behauptet, „fast ausnahmslos“ werde die Ungleichheit in Deutschland „weitaus höher eingeschätzt, als sie tatsächlich ist."'28 Die sozioökonomische Ungleichheit an den unterschiedlich hohen Einkommen von Armen und Reichen festzumachen, wie es Schröder tat, basierte jedoch auf einem Trugschluss und glich einem statistischen Taschenspielertrick. Denn man kann zwar Armut mit einem geringen Einkommen gleichsetzen, darf Reichtum aber nicht auf ein hohes Einkommen reduzieren. Vielmehr gehört zum Reichtum immer auch und sogar in allererster Linie ein großes (Kapital-)Vermögen.

Schröder wirft Karl Marx eine „kulturpessimistische Sichtweise" vor, weil dieser im Kommunistischen Manifest eine Verarmung des Proletariats vorausgesagt habe. Wenn man sieht, unter welchen Arbeitsbedingungen und zu welchen Löhnen transnationale Konzerne die Bewohner von Ländern der sogenannten Dritten und Vierten Welt billige Massenware für die Konsumtempel des globalen Nordens produzieren lassen, ist genau das eingetreten, was Marx und Engels befürchtet hatten: die zu ihrer Zeit in dem Maße noch kaum vorstellbare Bereicherung einer kleinen Schicht von Kapitaleigentümern bzw. Großaktionären durch brutale Ausbeutung und Verelendung einer riesigen Schar wehrloser Industriearbeiter. Dass jahrzehntelange Kämpfe der Arbeiter und Gewerkschafter nötig waren, um ähnliche Ver-

28 Vgl. M. Schröder: Warum die Gesellschaftswissenschaften keinen realitätsgetreuen Blick auf die Welt vermitteln, in: Gesellschaft - Wirtschaft - Politik (GWP), 68. Jg. (2019), H. 2, S. 237. 
hältnisse, wie sie bis heute in Ländern des globalen Südens existieren, im westlichen Nachkriegskapitalismus zu verhindern, verschweigt Schröder, obwohl die Lebensbedingungen von Millionen Beschäftigten des modernen Prekariats, von Paketboten, Fahrradkurieren und Mitarbeitern in deutschen Schlachtbetrieben, hieran durchaus erinnern.

\section{Ungleichheit wird systematisch unterschätzt}

Der Volkswirt und frühere Caritas-Generalsekretär Georg Cremer beklagt den angeblichen „Niedergangsdiskurs“ und behauptet demgegenüber, dass Deutschland gerechter sei, als wir meinen. Er führt zur Begründung seines Buchtitels hauptsächlich die „seit 2005 alles in allem erfolgreiche Arbeitsmarktpolitik" und die gestiegenen Sozialausgaben an. Letztere sind in den fünf Jahren vor und in den ersten beiden Jahren nach der Wirtschafts- und Finanzkrise 2008/2009 in Relation zur Entwicklung des Bruttoinlandsprodukts jedoch sogar gesunken und könnten angesichts ihres Rekordstandes während des Konjunktureinbruchs ebenso gut als Indiz für wachsende soziale Probleme gewertet werden. „Viel Geld für Sozialpolitik - das ist in erster Linie ein Armutszeugnis." ${ }^{29}$ Übrigens gaben mehrere EU-Staaten (Frankreich, Dänemark, Österreich, Italien und Schweden) mehr für Soziales aus.

Deutschland ist aus mindestens zwei Gründen sogar ungerechter, als Cremer glauben machen will und die meisten Bürger annehmen dürften: Erstens ist die Nichtinanspruchnahmequote, also der Anteil jener Personen, die aufgrund ihrer Armutslage staatliche Transferleistungen beantragen könnten, es aber nicht tun, sehr hoch. Sie beträgt bei der Grundsicherung für Arbeitsuchende (Hartz IV) ca. $50 \%$ und bei der Grundsicherung im Alter bis zu 68\%.30 Von zwei möglichen Beziehern des Arbeitslosengeldes II stellt also einer keinen Antrag und von drei armen Seniorinnen verzichten zwei auf die ihnen zustehenden Transferleistungen. Betroffene wissen oft schlicht nicht, dass es diese Sozialtransfers gibt oder dass sie einen Anspruch darauf hätten. Manche sind auch zu stolz, schämen sich oder scheuen

29 R. Dillmann, A. Schiffer-Nasserie: Der soziale Staat. Über nützliche Armut und ihre Verwaltung, Hamburg 2018, S. 250.

30 Vgl. I. Becker: Finanzielle Mindestsicherung und Bedürftigkeit im Alter, in: Zeitschrift für Sozialreform, 58. Jg. (2012), H. 2, S. 123; J. Friedrichsen, R. Schmacker: Die Angst vor Stigmatisierung hindert Menschen daran, Transferleistungen in Anspruch zu nehmen, in: DIWWochenbericht, 86. Jg. (2019), H. 26, S. 456. den Papierkrieg mit einer Behörde. Wieder andere fürchten deren Unterhaltsrückgriff auf ihre Verwandten.

Zweitens wird die Reichtumskonzentration in Deutschland wegen genereller Probleme mit Vermögensdaten und besonders ungenügender Informationen zum oberen Randbereich systematisch unterschätzt. Auch erfassen die verfügbaren Datenquellen die höchsten Einkommen entweder - wie die Einkommens- und Verbrauchsstichprobe des Statistischen Bundesamtes - aufgrund einer „Abschneidegrenze“ gar nicht oder sind gerade im obersten Bereich wegen Verschleierungstaktiken der Spitzenverdiener ziemlich ungenau. DIWMitarbeiter haben herausgefunden, dass auch Studien zu den Verteilungsrelationen auf der Basis von Bevölkerungserhebungen die Vermögensungleichheit in Deutschland tendenziell unterschätzen. ${ }^{31}$ Schließlich kann man auch nicht auf offizielle Zahlen des Staats zur Verteilung der Privatvermögen zurückgreifen, weil dieser selbst über keine Daten mehr verfügt, seitdem er die Erhebung der Vermögensteuer zum 1. Januar 1997 beendet und die Kapitalertragsteuer zum 1. Januar 2009 in eine pauschale Abgeltungsteuer in Höhe von $25 \%$ umgewandelt hat, die von den (Depot-)Banken erhoben und ohne persönliche Veranlagung der Steuerpflichtigen an die Finanzverwaltung weitergeleitet wird.

Je systemkonformer die Soziologie und je marktfixierter die Ökonomie in Deutschland wird, umso weniger sind diese beiden für das Problem der Ungleichheit „zuständigen“ Wissenschaften fähig, die Sozialstruktur der Gesellschaft zu entschlüsseln. Ihre genuine Funktion als nur der Wahrheit verpflichtete Fachdisziplinen ist aber nicht die Ideologieproduktion, sondern die Entmystifikation der bestehenden Verteilungs-, Macht- und Herrschaftsverhältnisse. Nur an der Oberfläche sind die Bürger demokratischer Staaten gleich, sei es bezüglich ihrer Rechte und politischen Einflussmöglichkeiten oder bezüglich ihrer Bildungs- und sozialen Aufstiegschancen. Schaut man genauer hin, entdeckt man krasse Formen der Ungleichheit, die sich nach wie vor in Klassen und Schichten materialisiert, wenngleich die Sozialstruktur heute differenzierter, komplexer und komplizierter zu durchschauen ist als zu Marxens Lebzeiten. ${ }^{32}$

31 Vgl. C. Westermeier, M. M. Grabka: Große statistische Unsicherheit beim Anteil der Top-Vermögenden in Deutschland, in: DIW-Wochenbericht, 82. Jg. (2015), H. 7, S. $123 \mathrm{ff}$.

32 Vgl. ausführlicher C. Butterwegge, a.a. O., S.217 ff.

Title: Economists Show Increasing Inequality the Cold Shoulder

Abstract: For some time now, growing inequality has been the cardinal problem of our society, if not of all humanity. While on a global scale this results in crises and wars, leading in turn to major migratory movements, in this country it has led to a threat to cohesion and representative democracy. Nevertheless, most German economists have never seriously addressed the problem of inequality and have never looked for ways to solve it. Rather, they have ignored the problem or negated or relativized its significance.

JEL Classification: B10, B20, D63 